\title{
Polycaprolactone-Chitin Nanofibrous Mats as Potential Scaffolds for Tissue Engineering
}

\author{
Min Sup Kim, Sang Jun Park, Bon Kang Gu, and Chun-Ho Kim \\ Laboratory of Tissue Engineering, Korea Institute of Radiological and Medical Science, Seoul 139-240, Republic of Korea \\ Correspondence should be addressed to Chun-Ho Kim, chkim@kcch.re.kr
}

Received 16 December 2011; Revised 29 February 2012; Accepted 14 March 2012

Academic Editor: Tong Lin

Copyright ( $) 2012$ Min Sup Kim et al. This is an open access article distributed under the Creative Commons Attribution License, which permits unrestricted use, distribution, and reproduction in any medium, provided the original work is properly cited.

\begin{abstract}
We describe here the preparation of poly(caprolactone) (PCL)-chitin nanofibrous mats by electrospinning from a blended solution of PCL and chitin dissolved in a cosolvent, 1,1,1,3,3,3-hexafluoro-2-propanol and trifluoroacetic acid. Scanning electron microscopy showed that the neutralized PCL-chitin nanofibrous mats were morphologically stable, with a mean diameter of $340.5 \pm 2.6 \mathrm{~nm}$, compared with a diameter of $524.2 \pm 12.1 \mathrm{~nm}$ for PCL mats. The nanofibrous mats showed decreased water contact angles as the proportion of chitin increased. However, the tensile properties of nanofibrous mats containing $30 \sim 50 \%$ $(\mathrm{wt} / \mathrm{wt})$ chitin were enhanced compared with PCL-only mats. In vitro studies showed that the viability of human dermal fibroblasts (HDFs) for up to 7 days in culture was higher on composite (OD value: $1.42 \pm 0.09$ ) than on PCL-only $(0.51 \pm 0.14)$ nanofibrous mats, with viability correlated with chitin concentration. Together, our results suggest that PCL-chitin nanofibrous mats can be used as an implantable substrate to modulate HDF viability in tissue engineering.
\end{abstract}

\section{Introduction}

Scaffold development is critical for the success of tissue regeneration. Specifically, scaffolds should be composed of biocompatible and biodegradable materials and exhibit mechanical properties similar to those of target tissue $[1,2]$. Moreover, the scaffold should have structural and chemical properties closely mimicking those of the extracellular matrix (ECM), allowing them to modulate appropriate cellular behavior such as adhesion, migration, proliferation, and differentiation [3-5].

Among existing methods for the fabrication of scaffolds, electrospinning techniques provide unique benefits that satisfy these crucial requirements, including simplicity in imitating nanofibrous mats with three-dimensional nanoscaled structures of native ECM and versatility in the choice of biocompatible polymers [6-8]. In particular, various types of nanofibrous mats can be fabricated from blends of synthetic and natural polymers by a choice of suitable solvents. As synthetic polymer, for examples, poly(caprolactone) (PCL) can be modulated with respect to molecular weight, degradation rate, and mechanical properties; however, they lack specific recognition sites for cell, and therefore PCL scaffolds often exhibit poor cell adhesion, migration, and proliferation [9-13]. In contrast, the nanofibrous mats made of natural polymers such as chitin offer several advantages, including being nonimmunogenic, biocompatible, and biodegradable, and possessing hemostatic properties. These mats, however, have poor mechanical properties. In addition, it is difficult to prepare nanofibrous forms of chitin, due to its poor solubility in common organic solvents [1417]. Therefore, chitin/poly (glycolic acid) (PGA) nanofibrous mats, prepared by blending in 1,1,1,3,3,3-hexafluoro-2propanol (HFP), have been investigated in the fabrication of biodegradable, nanofibrous scaffolds for tissue engineering [18]. Since native chitin is not readily dissolved in HFP, nanofibrous forms are frequently prepared by high-dose gamma irradiation for over 10 days to enhance its solubility (to reduce its molar mass) [19]. This method, however, is time consuming and expensive, indicating the need for an easier, less-expensive, and energy-saving method to prepare chitin solutions in the fabrication of a broad range of chitin based nanofibers.

To solve these problems, we prepared a solution of PCL and chitin dissolved in a solvent composed of HFP and trifluoroacetic acid (TFA) within 2 days. And then 
PCL-chitin blended nanofibrous mats were fabricated using electrospinning process. However, chitosan-based nanofibers from TFA-based solvents lost their fibrous structure in aqueous conditions and required neutralized process for elimination of trifluoroacetate salts in nanofibers [15]. We especially investigated morphological stability of nanofibrous mats in aqueous conditions before and after neutralized process using sodium carbonate solution. And then, we investigated the effect of chitin concentration on the morphology, hydrophilicity, and mechanical properties of the nanofibrous mats. We also evaluated the effect of chitin content on the adhesion and proliferation of human dermal fibroblasts (HDFs) on PCL-chitin nanofibrous mats.

\section{Materials and Methods}

2.1. Materials. PCL (molecular weight $=80,000$ ), sodium carbonate, and TFA were purchased from Sigma-Aldrich (St. Louis, MO, USA). Chitin ( $\beta$-form, average molecular weight $=612 \mathrm{kDa}$, deacetylation degree $=32.4 \%)$ was kindly provided by ARABIO (Seoul, Korea). HFP (HPLC grade) was from Tokyo Chemical Industry (Kita-Ku, Tokyo, Japan), and ethanol (absolute for analysis) was from Merck (Darmstadt, Germany). Dulbecco's modified Eagle's medium (DMEM) with low glucose, Dulbecco's phosphate-buffered saline (DPBS), and $0.05 \%$ trypsin-EDTA were purchased from Gibco BRL (Carlsbad, CA, USA), and fetal bovine serum (FBS) and penicillin-streptomycin (PS) were from WelGene Inc. (Daegu, Korea). Cell counting kit-8 (CCK-8) was purchased from Dojindo Laboratories (Kumamoto, Japan), and albumin Fraction V (from bovine blood, BSA) was from Amersham/USB (Arlington Heights, IL, USA).

2.2. Viscosity of Polymeric Solutions. PCL 5\% (wt/v) was dissolved in HFP, and chitin 5\% (wt/v) was dissolved in HFP:TFA (volume ratio $=9: 1$ ) for 1 day. A series of PCL: chitin blends with volume ration of $100: 0,70: 30$, $50: 50,30: 70$, and $0: 100$ (PCL, P7C3, P5C5, P3C7, and chitin, resp.) were prepared by thorough stirring for 1 day. The viscosity of each solution was determined using an AR 2000 EX rheometer (TA instrument, New Castle, DE, USA) using a cone and plate geometry of $4 \mathrm{~cm}$ diameter and $1^{\circ}$ cone angle. Each solution was injected into a rotational cone and plate at a controlled shear rates, and the viscosity of each was measured at $10^{\circ} \mathrm{C}$ for 3 minutes with a constant shear stress of $1 \mathrm{~Pa}$.

2.3. Preparation of Nanofibrous Mats. Following the conventional electrospinning procedure, each polymer solution was injected from a syringe through a stainless-steel needle using a syringe pump (KD Scientific, Holliston, MA, USA) at $1 \mathrm{~mL} / \mathrm{hr}$. Using a high-voltage power supply (Wookyung Tech, Incheon, Korea), $15 \mathrm{kV}$ was applied between the syringe needle and a grounded collector, with the latter placed $15 \mathrm{~cm}$ below the tip of the needle to collect the nanofibers. The resulting nanofibrous mats were dried overnight at room temperature.
2.4. Neutralized Treatments of Nanofibrous Mats. Chitincontained nanofibrous mats were neutralized to eliminate trifluoroacetate salts for morphological stability of nanofibers. After drying at room temperature, the P7C3, P5C5, and P3C7 samples were soaked in 99.9\% ethanol for 30 minutes, neutralized by immersion in $0.1 \mathrm{M}$ sodium carbonate dissolved in $70 \%$ ethanol for 30 minutes, rinsed sequentially with $50 \%$ ethanol and deionized water, and lyophilized at $-80^{\circ} \mathrm{C}$ under vacuum ( 5 mTorr) for 1 day. Without neutralized treatments, PCL samples were soaked in $70 \%$ ethanol for 30 minutes, rinsed sequentially deionized water, and lyophilized. Finally, the resulting all nanofibrous mats were dried in vacuum at room temperature prior to further characterization.

2.5. Morphological Characterization. To investigate of the morphological stability nanofibers, nonneutralized and neutralized P5C5 samples were incubated in $37^{\circ} \mathrm{C}$ PBS buffer solution for 3 hours, rinsed deionized water, and lyophilized. The resulting samples were mounted onto stubs and coated with gold using a sputter coater (Eiko IB3; Tokyo, Japan). The morphology of the nanofibers was investigated using a MIRA II field emission-scanning electron microscope (FE-SEM, Tescan, Libusinatr, Czech Republic). Moreover, the neutralized nanofibrous mats were compared with nonneutralized samples to investigate morphological change of nanofibers after neutralization process using the FE-SEM. And ten fields from each image were selected, and the fiber diameters were manually measured using a ruler generated within the image analyzer (Image Pro-Plus; Media Cybernetics, Inc., MD, USA) and averaged.

2.6. Fourier Transform-Infrared Analysis. The chemical integrity of the nanofibrous mats was investigated by Fourier transform-infrared (FTIR) spectroscopy (Tensor 27; Bruker Optics, Ettlingen, Germany). About $5 \mathrm{mg}$ of each sample was cut into small pieces to prepare $\mathrm{KBr}$ discs, and FTIR spectra from 4000 to $500 \mathrm{~cm}^{-1}$ were recorded.

2.7. Water Contact Angle. The hydrophilicity of the nanofibrous mats was assessed by a contact angle goniometer (Phoenix 150; SEO, Seoul, Korea). Deionized water $(5 \mu \mathrm{L})$ was dropped onto the flat surface of mats, and the water contact angle was calculated after stabilization for 5 seconds.

2.8. Tensile Properties of Nanofibrous Mats. The tensile properties of the nanofibrous mats were characterized using a tabletop uniaxial testing machine (Instron 5567; Canton, MA, USA) with a $10-\mathrm{N}$ load cell under a crosshead speed of $1 \mathrm{~mm} / \mathrm{min}$ and ambient conditions. All samples were fixed in the form of a rectangle of dimensions $40 \times 10 \mathrm{~mm}$.

2.9. In Vitro Evaluation of Nanofibrous Mats. To study cellular responses to the nanofibrous mats, HDFs (MCTT, Seoul, Korea) were cultured in DMEM supplemented with $10 \%$ FBS and $1 \%$ PS under standard culture conditions $\left(37^{\circ} \mathrm{C}\right.$, $5 \% \mathrm{CO}_{2}$ ). Circular nanofibrous mats (diameter: $15 \mathrm{~mm}$ ) prepared by punchingout were sterilized with $70 \%$ ethanol 


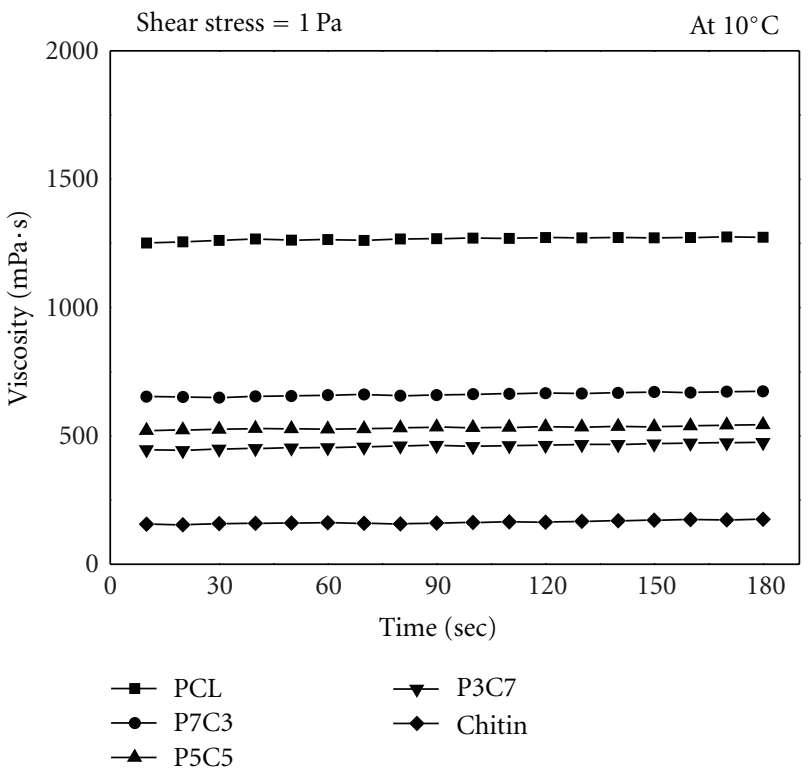

(a)

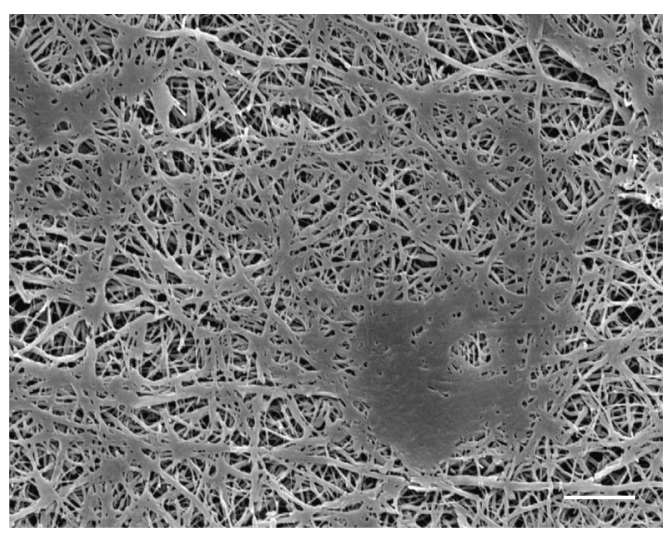

(b)

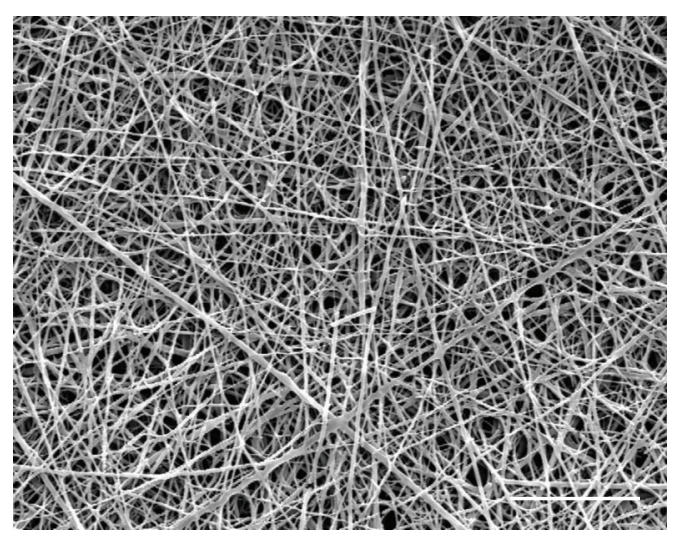

(c)

FIgURE 1: (a) Viscosity of 5\% (wt/v) PCL, chitin and their blends with volume ratios of PCL: chitin of 100:0, 70:30, 50:50, and 30:70 (PCL, P7C3, P5C5, and P3C7 solutions, resp.). The viscosity of polymeric solutions was determined at $10^{\circ} \mathrm{C}$ with constant shear stress of $1 \mathrm{~Pa}$. SEM images of (b) nonneutralized and (c) neutralized P5G5 nanofibrous mats immersing in PBS at $37^{\circ} \mathrm{C}$ for 3 hours. Scale bar is $10 \mu \mathrm{m}$.

and UV irradiation. HDFs (passage $=9$ ) at approximately $70 \%$ confluency were enzymatically lifted using trypsinEDTA and seeded onto the mats at a density of $5 \times$ $10^{3}$ cells $/ \mathrm{cm}^{2}$. The viability of these HDFs was evaluated at 1 , 4 , and 7 days using CCK- 8 assays. Cell-seeded nanofibrous mats $(n=4)$ were rinsed with DPBS, and $100 \mu \mathrm{L}$ CCK-8 solution was added to each sample. The optical density (OD) of each sample was measured at $450 \mathrm{~nm}$ using a plate reader (Spectra Max M2e; Molecular Devices, Sunnyvale, CA, USA).

At 1 and 7 days after seeding, HDFs cultured on nanofibrous mats were incubated with Alexa Fluor 488 phalloidin (Invitrogen) and 4',6-diamidino-2-phenylindole (DAPI, Invitrogen) to stain for actin filaments and nuclei, respectively. The cells were examined by confocal laser scanning microscopy (CLSM, C1; Nikon Corp., Chiyoda-ku, Japan).
2.10. Statistical Analysis. Quantitative data were obtained in triplicate and are reported as means \pm standard deviations, where indicated. Statistical analyses were performed using a one-way analysis of variation (ANOVA), followed by Tukey HSD for multiple comparisons. A $P$ value $<0.05$ was considered statistically significant.

\section{Results and Discussion}

In electrospinning process, viscosity of the polymer solutions was critical in generating nanofibers with uniform diameter, with lower viscosity associated with decreased fiber diameter [20-22]. As preliminary experiment, we investigated the viscosity of PCL, chitin, and their blended solutions using a rheometer. We found that the viscosity of PCL 


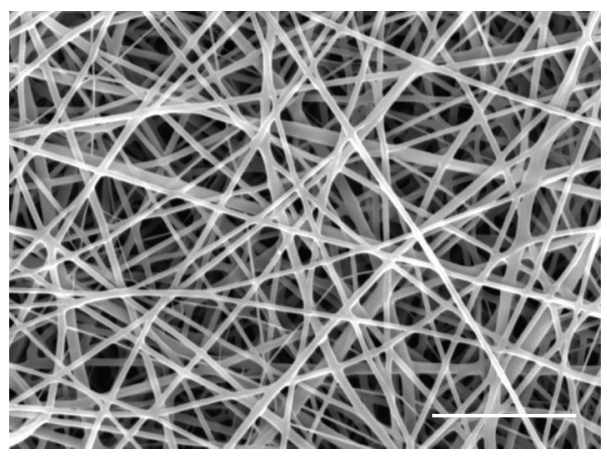

(a)

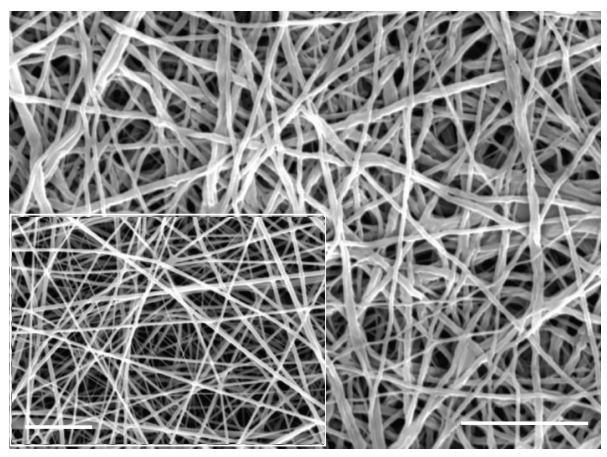

(c)

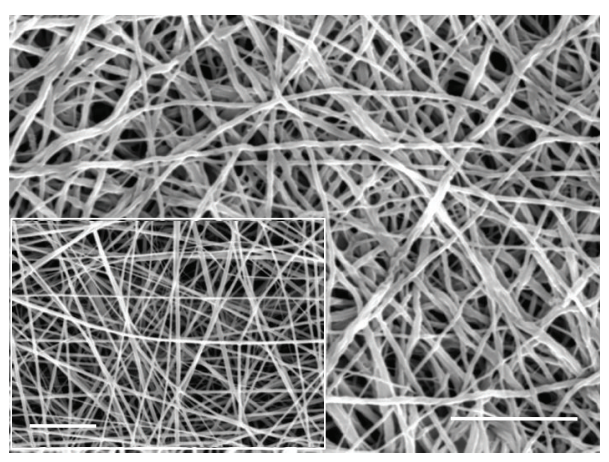

(b)

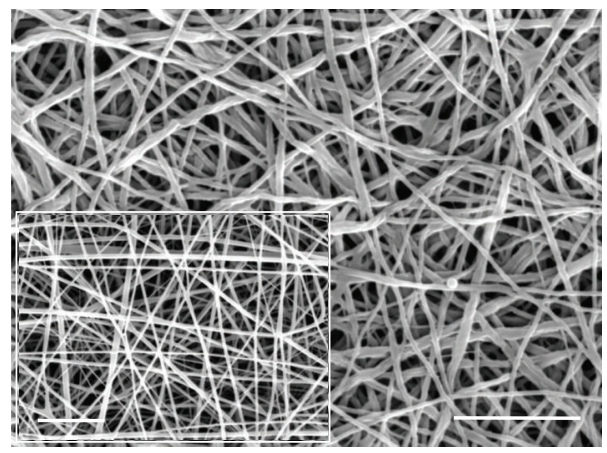

(d)

FIgURE 2: Representative SEM images of (a) PCL nanofibrous mats, and neutralized PCL-chitin nanofibrous mats; (b) P7C3, (c) P5C5, and (d) P3C7, respectively. Inset SEM images in smaller boxes show corresponding nonneutralized samples. Scale bar is $5 \mu \mathrm{m}$.

solution was $1267.27 \pm 6.68 \mathrm{mPa} \cdot \mathrm{s}$, and the viscosity of chitin solution was $163.83 \pm 6.51 \mathrm{mPa} \cdot \mathrm{s}$, with the viscosity of blended PCL-chitin solutions decreasing as the ratio of chitin increased, with P7C3, P5C5 and P3C7 solutions having viscosities of $661.72 \pm 7.34,531.88 \pm 6.22$, and $460.44 \pm 9.43 \mathrm{mPa} \cdot \mathrm{s}$, respectively (Figure $1(\mathrm{a})$ ). From these preliminary results, we therefore prepared PCL and PCLchitin nanofibrous mats using the electrospinning method (Figure 2). Under our fixed condition, chitin-only nanofibers could not be generated due to the low viscosity of the chitin solution.

For in vitro and vivo application of nanofibrous mats, we examined their morphological stability of PCL-chitin samples under aqueous conditions. SEM images of nonneutralized P5C5 nanofibrous mats show that the nanofibrous structure had collapsed due to hydrolysis or dissolution of $\mathrm{NH}_{3}^{+} \mathrm{CF}_{3} \mathrm{COO}^{-}$salt residues (by chitin dissolved in TFA) within the nanofibers (Figure 1(b)) [23]. However, this collapse was no longer observed at neutralized nanofibrous mats (Figure $1(\mathrm{c})$ ) after incubation in $37^{\circ} \mathrm{C}$ PBS buffer solution for 3 hours, suggesting the need for a neutralization process, eliminating salt residues, to maintain the nanofibrous structure under aqueous conditions.

SEM images of PCL-chitin nanofibrous mats before and after neutralization in $0.1 \mathrm{M}$ sodium carbonate dissolved in $70 \%$ ethanol showed that, after neutralization, the nanofibers maintained a uniform and interconnected nanofibrous structure (Figure 2). These randomly oriented nanofibers formed three-dimensional open pores that were homogeneously distributed throughout the structure. Although this neutralization process seemed to have minimal effect on the overall morphology of the fibers, neutralization caused the fibers to make closer contact with each other and resulted in a more deformed structure, particularly at fiber-fiber junctions. Collectively, these results suggest that PCL-chitin nanofibrous mats require neutralization and that their reaction with sodium carbonate dissolved in ethanol effectively prevented the rapid dissolution of chitin from these nanofibrous mats.

To quantitatively analyze the effect of neutralization on the morphology of nanofibers, we measured their average diameters from SEM images. We found that the mean diameter of PCL nanofibers was $524.2 \pm 12.1 \mathrm{~nm}$, and that the mean diameters of blended nanofibers decreased as the concentration of chitin increased (Table 1). Similarly, the mean diameter of chitin/silk fibroin (SF) nanofibers decreased from 920 to $340 \mathrm{~nm}$, as the concentration of chitin increased [24]. We also found that the average diameters of PCL-chitin nanofibers increased slightly after neutralization, for example, from $262.6 \pm 15.9 \mathrm{~nm}$ to $341.8 \pm 22.4 \mathrm{~nm}$ for P5C5 nanofibers. This may be due to the presence of a tiny amount of water, resulting in partial hydrolysis or the dissolution of a soluble component during neutralization $[15,25]$.

FTIR analysis of neutralized P5C5 nanofibrous mats showed peaks at 2949, 2865, and $1720 \mathrm{~cm}^{-1}$, characteristic of 


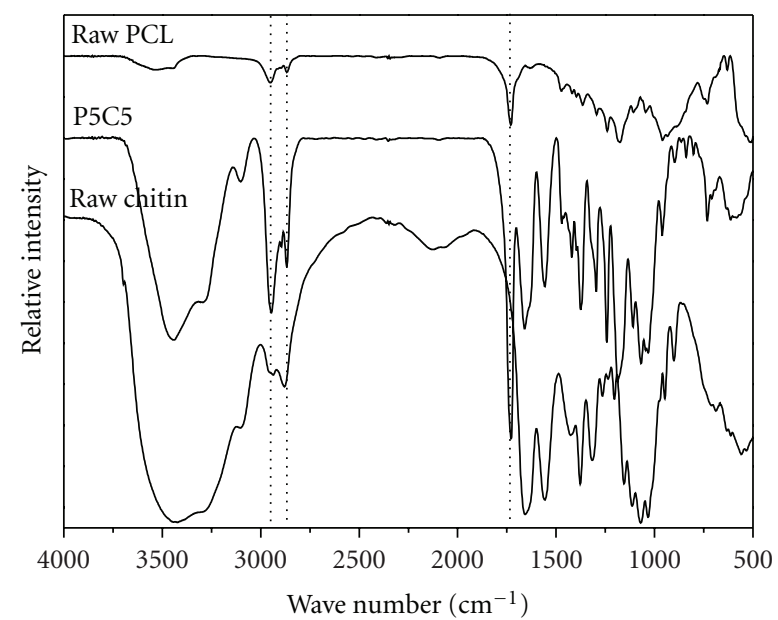

(a)
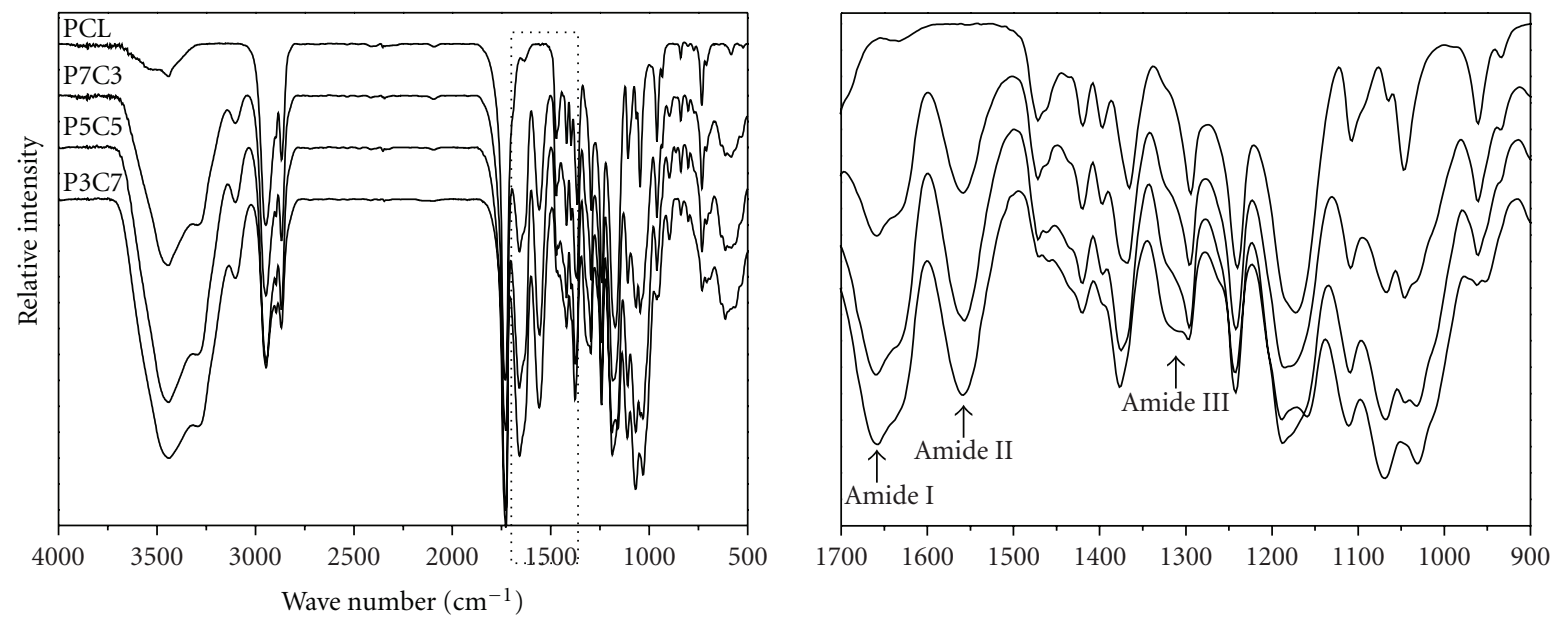

(b)

FIGURE 3: FTIR analysis of (a) raw material and P5C5 nanofibrous mats and (b) PCL, and neutralized PCL-chitin nanofibrous mats.

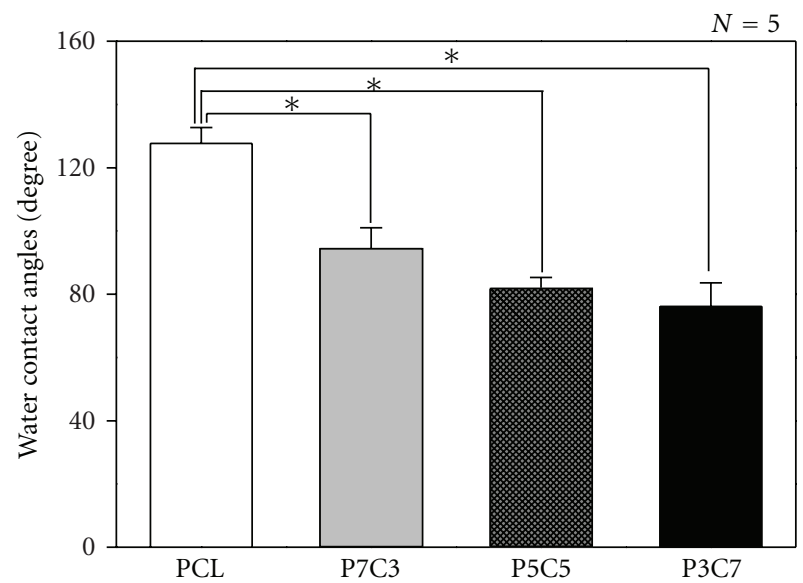

FIGURE 4: Water contact angles on the surfaces of PCL and neutralized PCL-chitin nanofibrous mats. Deionized water $(5 \mu \mathrm{L})$ was dropped onto the flat surface of mats, and the contact angle was calculated after stabilization for 5 seconds.
TABLE 1: Average fiber diameters of PCL and PCL-chitin composite nanofibers $N=50$.

\begin{tabular}{lcc}
\hline Sample & $\begin{array}{c}\text { Before } \\
\text { Neutralization }(\mathrm{nm})\end{array}$ & $\begin{array}{c}\text { After } \\
\text { Neutralization }(\mathrm{nm})\end{array}$ \\
\hline PCL & $524.2 \pm 12.1$ & - \\
P7C3 & $263.4 \pm 16.4$ & $337.5 \pm 19.4$ \\
P5C5 & $262.6 \pm 15.9$ & $341.8 \pm 22.4$ \\
P3C7 & $258.8 \pm 19.7$ & $342.4 \pm 21.6$ \\
\hline
\end{tabular}

asymmetric and symmetric stretching of $\mathrm{CH}_{2}$ and carbonyl stretching of PCL (Figure 3(a)), as well as peaks at $3479(\mathrm{OH}$ stretching), 1656, 1556, and 1314 (amides I, II, and III) $\mathrm{cm}^{-1}$ characteristic of raw chitin [16]. All samples showed the peaks characteristic of PCL (Figure 3(b)), with the relative intensity of peaks characteristic of chitin characteristic (e.g., amide I, II, and III) increasing as the concentration of chitin increased. 


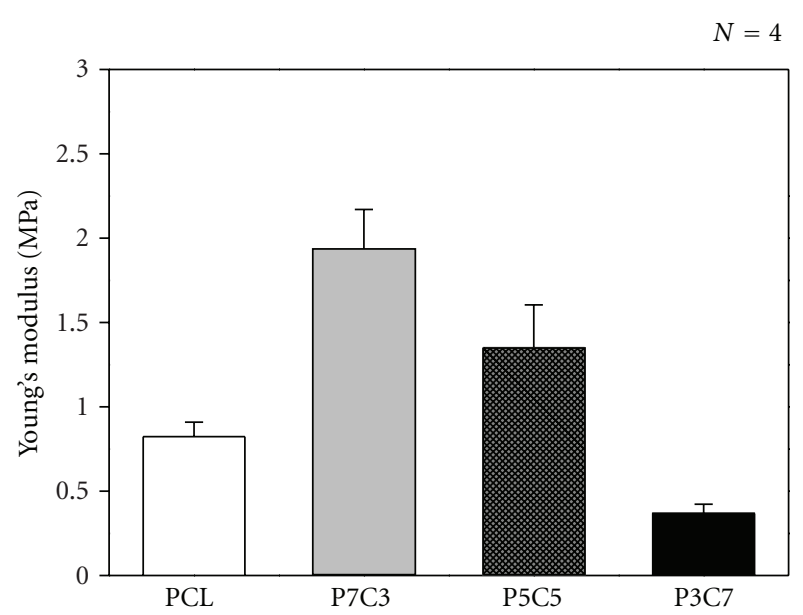

(a)

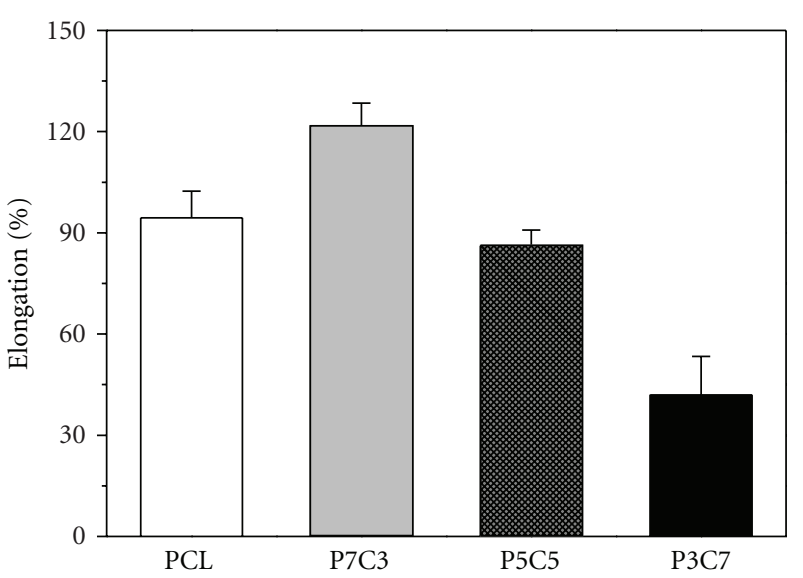

(b)

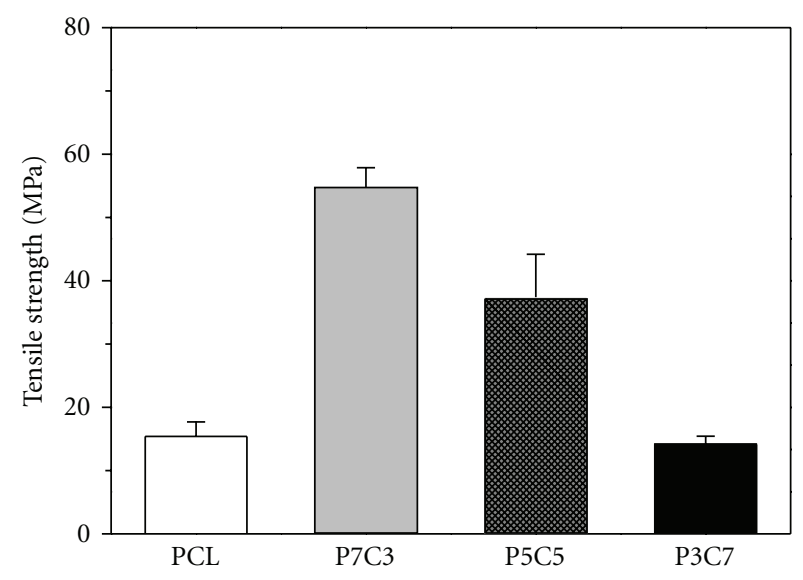

(c)

Figure 5: Mechanical properties of PCL and neutralized PCL-chitin nanofibrous mats; (a) Young's modulus, (b) elongation, and (c) tensile strength.

When we assessed the water contact angles of PCL and neutralized PCL-chitin nanofibrous mats, we found that the PCL samples were moderately hydrophobic with a contact angle of $127.6 \pm 5.1^{\circ}$ (Figure 4). Incorporation of chitin increased the hydrophilicity of the nanofibrous mats, with contact angles of $\mathrm{P} 7 \mathrm{C} 3, \mathrm{P} 5 \mathrm{C} 5$, and $\mathrm{P} 3 \mathrm{C} 7$ decreasing to $94.4 \pm 6.6,81.6 \pm 3.7$, and $76.1 \pm 7.5^{\circ}$, respectively, due to the increased presence of hydrophilic functional groups, such as hydroxy and amide groups, in chitin. This result indicates that homogeneously mixed chitin and PCL results in hydrophilic nanofibrous mats, with the hydrophilicity easily modulated by adjusting the amount of chitin.

We also measured the Young's modulus, elongation at break, and tensile strength of the nanofibrous mats using a uniaxial testing machine (Figure 5). We found that PCL-only samples had a Young's modulus of $0.8 \pm 0.1 \mathrm{MPa}$, an elongation of $94.4 \pm 7.9 \%$, and a tensile strength of $15.4 \pm 2.3 \mathrm{MPa}$. In comparison, the mechanical properties of PCL-chitin blended nanofibrous mats were enhanced, with P7C3, P5C5, and P3C7 fibers having Young's modulus of $1.9 \pm 0.2,1.3 \pm 0.2$,

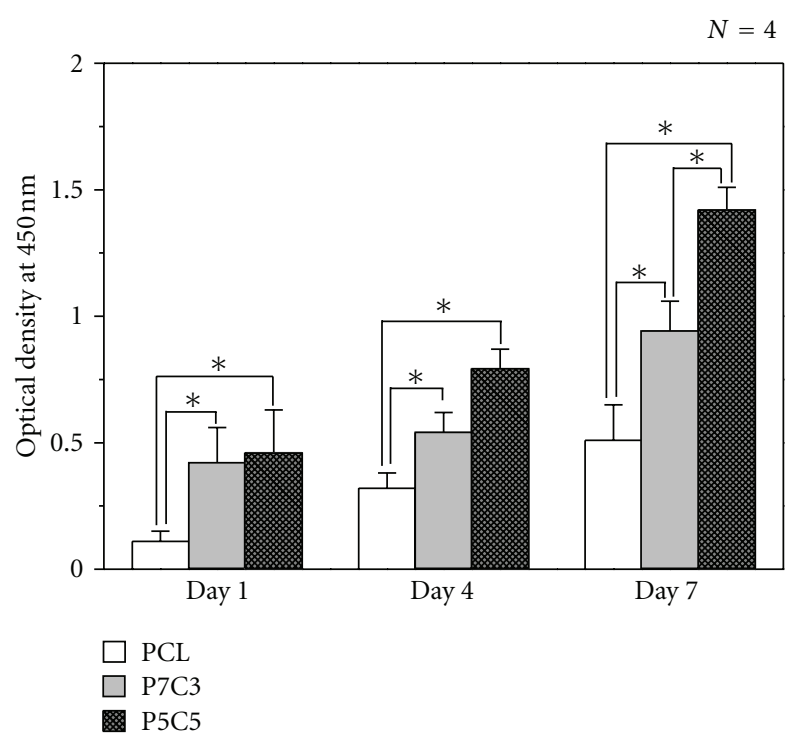

FIGURE 6: The viability of human dermal fibroblasts (HDFs) on PCL and PCL-chitin nanofibrous mats during 7 days. 


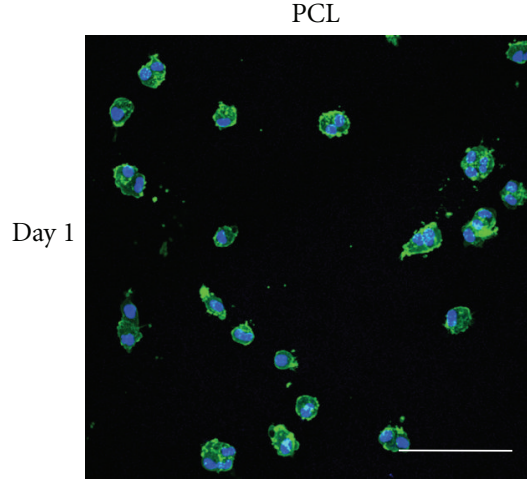

(a)

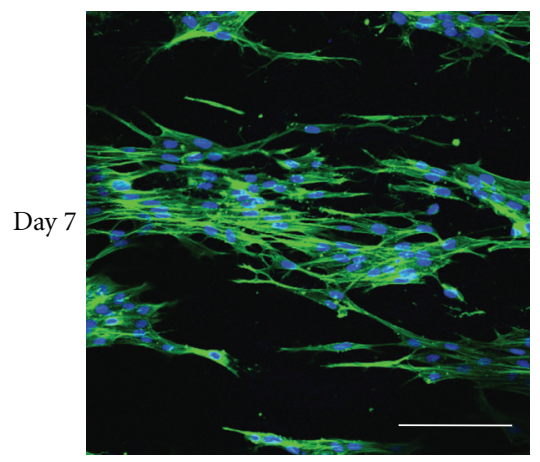

(d)

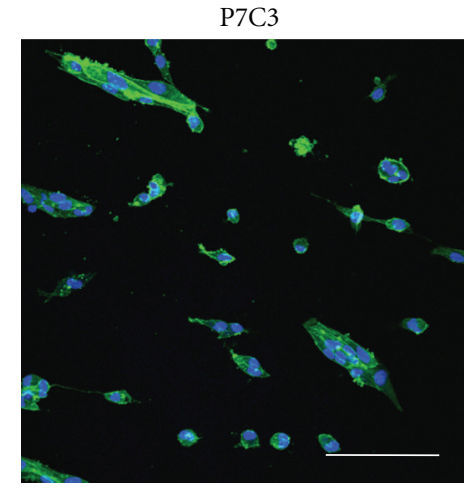

(b)

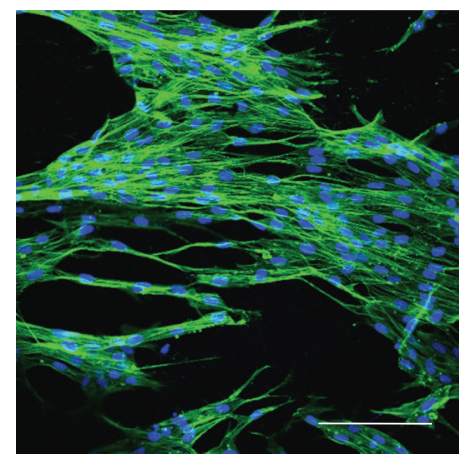

(e)

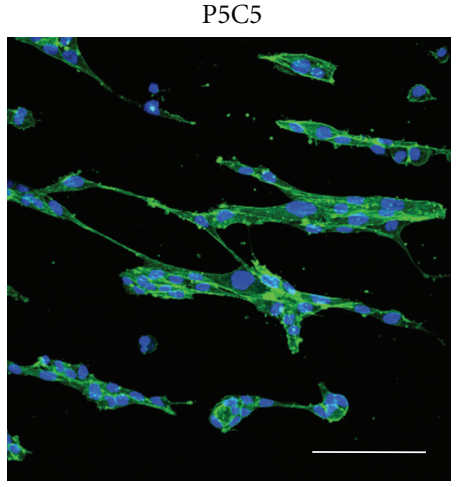

(c)

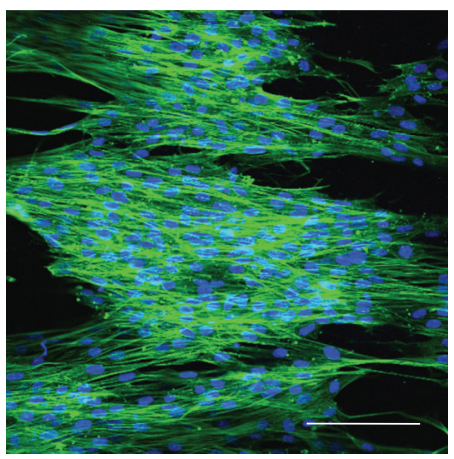

(f)

Figure 7: CLSM images of HDFs on PCL and PCL-chitin nanofibrous mats after 7 days of cultivation (scale bear is $100 \mu \mathrm{m})$. CLSM images of HDFs on (a) PCL, (b) P7C3, and (c) P5C5 nanofibrous mats on day 1, respectively. And HDFs on (d) PCL, (e) P7C3, and (f) P5C5 nanofibrous mats on day 7, respectively. Cell nuclei (blue) and actin filaments (green) were stained by DAPI and Alexa Fluor 488 phalloidin. Scale bar is $100 \mu \mathrm{m}$.

and $0.3 \pm 0.1 \mathrm{MPa}$, respectively, elongations of $121.7 \pm 6.7$, $86.1 \pm 4.7$, and $41.9 \pm 11.4 \%$, respectively, and tensile strengths of $64.7 \pm 2.8,34.1 \pm 6.8$, and $14.1 \pm 1.3 \mathrm{MPa}$, respectively. The enhanced tensile properties of PCL-chitin nanofibrous mats containing $30 \sim 50 \%(\mathrm{v} / \mathrm{v})$ chitin may be due to chitin acting as a rigid filler, with favorable compatibility with PCL only within a certain concentration range. Thus, $30 \%(\mathrm{v} / \mathrm{v})$ may be the maximum chitin concentration for reinforcing the mechanical properties of nanofibrous mats. The mechanical properties of PCL-chitin nanofibrous mats may be within clinically relevant ranges for the regeneration of human skin, since human skin has a Young's modulus of $83.3 \pm 34.9 \mathrm{MPa}$, a tensile strength of $21.6 \pm 8.4 \mathrm{MPa}$, and an elongation of $54.0 \pm 17.0 \%[26,27]$.

The appropriate method to measure the mechanical properties of nanofibrous mats is unclear because their average diameters, the direction of individual fibers, and their junctions/bonding may alter the results. Although our results are in part sufficient to show the bulk properties of nanofibrous mats, it seemed to be an optimum blending ratio (30 50\% chitin to PCL) of PCL-chitin nanofibrous mats.

We therefore investigated the proliferation of HDFs on PCL and neutralized PCL-chitin nanofibrous mats using the CCK-8 assay. As shown in Figure 6, we found that the OD values of PCL, P7C3, and P5C5 nanofibrous mats were $0.11 \pm$ $0.04,0.42 \pm 0.14$, and $0.46 \pm 0.17$, respectively, after 1 day of culture. From day 1 to 4 , the OD values of these groups increased as the chitin concentration increased, indicating that cell proliferation increased with chitin concentration. On day 7 , the OD value of P5C5 groups $(1.42 \pm 0.09)$ was higher than for PCL $(0.51 \pm 0.14)$ and P7C3 $(0.94 \pm$ 0.12). HDFs cultured on P7C 3 and P5C5 nanofibrous mats exhibited greater proliferation than cells cultured on PCL mats for up to 7 days, suggesting that the hydrophilic functional group of chitin may contribute to proliferation. Similarly, HDFs and normal human epidermal keratinocytes cultured on chitin-containing nanofibrous mats have higher viability than those cultured on synthetic polymers only due to the presence of biofunctional groups in chitin [24].

We confirmed the effects of chitin concentration in nanofibrous mats on the proliferation of cultured HDFs using immune fluorescence staining. HDFs cultured on PCLchitin nanofibrous mats for 1 day showed a more spreadout morphology and stable adhesion compared with the round shape of cells cultured on PCL alone (Figures 7(b) and $7(\mathrm{c})$ ). Moreover, cells cultured on blended nanofibrous mats were at much higher density with mature formation of actin filaments. On day 7, HDFs cultured on PCL nanofibrous mats (Figure $7(\mathrm{~d})$ ) had thinner spindle morphology 
than cells cultured on PCL-chitin samples. These cellular morphologies correlated with results of cellular viability (Figure 6). The enhanced cellular adhesion and proliferation on nanofibrous mats incorporating chitin may be due to the presence in chitin of hydrophilic functional groups, such as hydroxy and amide groups [16, 28, 29]; these hydrophilic groups have superior binding affinity compared with PCL alone, which shows poor cell adhesion and proliferation [12]. Our in vitro results indicate that alterations of chitin concentration in chitin-incorporating nanofibrous mats can modulate the proliferation of HDFs. These chitin-containing nanofibrous mats may induce more rapid HDF proliferation through stable adhesion and may have potential applications in tissue engineering.

\section{Conclusion}

We have described the preparation of PCL-chitin nanofibrous mats by electrospinning from a solution of PCL and chitin dissolved in a cosolvent of HFP and TFA. These blended nanofibrous mats showed greater morphological stability in aqueous condition after than before neutralization. Alterations in chitin concentration altered the hydrophilicity and tensile properties of PCL-chitin nanofibrous mats, factors crucial in their use as implantable substrates for in vitro culture of cells, as well as modulating the adhesion and proliferation of HDFs. Collectively, nanofibrous mats incorporating chitin may hold promise as functional nanofibrous scaffolds, which, in combination with therapeutic drugs, cytokines and peptides, may act as implants to control biological functions during tissue regeneration.

\section{Acknowledgments}

This work was supported by Industrial Strategic Technology Development Program (Multifunctional medical fiber complex technology), funded by the Ministry of Knowledge Economy (MKE, Korea) and by Nuclear Research Development Program of the Korea Science and Engineering Foundation (KOSEF) grant and funded by Ministry of Education, Science and Technology (MEST, Korea). The authors thank Professor Jong-Il Kim (Department of Food and Microbial Technology, Seoul Women's University, Seoul, Korea) for his invaluable help with the SEM analysis.

\section{References}

[1] M. E. Furth, A. Atala, and M. E. van Dyke, "Smart biomaterials design for tissue engineering and regenerative medicine," Biomaterials, vol. 28, no. 34, pp. 5068-5073, 2007.

[2] P. X. Ma, "Biomimetic materials for tissue engineering," Advanced Drug Delivery Reviews, vol. 60, no. 2, pp. 184-198, 2008.

[3] M. Radisic, H. Park, F. Chen et al., "Biomimetic approach to cardiac tissue engineering: oxygen carriers and channeled scaffolds," Tissue Engineering, vol. 12, no. 8, pp. 2077-2091, 2006.
[4] F. T. Moutos, L. E. Freed, and F. Guilak, "A biomimetic threedimensional woven composite scaffold for functional tissue engineering of cartilage," Nature Materials, vol. 6, no. 2, pp. 162-167, 2007.

[5] G. C. Engelmayr, M. Cheng, C. J. Bettinger, J. T. Borenstein, R. Langer, and L. E. Freed, "Accordion-like honeycombs for tissue engineering of cardiac anisotropy," Nature Materials, vol. 7, no. 12, pp. 1003-1010, 2008.

[6] C. P. Barnes, S. A. Sell, E. D. Boland, D. G. Simpson, and G. L. Bowlin, "Nanofiber technology: designing the next generation of tissue engineering scaffolds," Advanced Drug Delivery Reviews, vol. 59, no. 14, pp. 1413-1433, 2007.

[7] T. J. Sill and H. A. von Recum, "Electrospinning: applications in drug delivery and tissue engineering," Biomaterials, vol. 29, no. 13, pp. 1989-2006, 2008.

[8] H. N. Park, J. B. Lee, and I. K. Kwon, "Electrospun nanofibers using biomacromolecules and their applications as tissue engineered scaffold," International Journal of Tissue Regeneration, vol. 1, pp. 10-20, 2010.

[9] S. H. Oh, I. K. Park, J. M. Kim, and J. H. Lee, "In vitro and in vivo characteristics of PCL scaffolds with pore size gradient fabricated by a centrifugation method," Biomaterials, vol. 28, no. 9, pp. 1664-1671, 2007.

[10] L. Shor, S. Güçeri, X. Wen, M. Gandhi, and W. Sun, "Fabrication of three-dimensional polycaprolactone/hydroxyapatite tissue scaffolds and osteoblast-scaffold interactions in vitro," Biomaterials, vol. 28, no. 35, pp. 5291-5297, 2007.

[11] M. E. Hoque, W. Y. San, F. Wei et al., "Processing of polycaprolactone and polycaprolactone-based copolymers into $3 \mathrm{D}$ scaffolds, and their cellular responses," Tissue Engineering A, vol. 15, no. 10, pp. 3013-3024, 2009.

[12] B. W. Tillman, S. K. Yazdani, S. J. Lee, R. L. Geary, A. Atala, and J. J. Yoo, "The in vivo stability of electrospun polycaprolactonecollagen scaffolds in vascular reconstruction," Biomaterials, vol. 30, no. 4, pp. 583-588, 2009.

[13] D. Lee, Y. Song, C. Kim et al., "Effect of starch-polycaprolactone scaffolds on the attachment, proliferation, and phenotypes of olfactory ensheathing cells," International Journal of Tissue Regeneration, vol. 1, pp. 21-28, 2010.

[14] I. Y. Kim, S. J. Seo, H. S. Moon et al., "Chitosan and its derivatives for tissue engineering applications," Biotechnology Advances, vol. 26, no. 1, pp. 1-21, 2008.

[15] R. Jayakumar, M. Prabaharan, S. V. Nair, and H. Tamura, "Novel chitin and chitosan nanofibers in biomedical applications," Biotechnology Advances, vol. 28, no. 1, pp. 142-150, 2010.

[16] R. Jayakumar, K. P. Chennazhi, S. Srinivasan, S. V. Nair, T. Furuike, and H. Tamura, "Chitin scaffolds in tissue engineering," International Journal of Molecular Sciences, vol. 12, no. 3, pp. 1876-1887, 2011.

[17] R. A. A. Muzzarelli, J. Boudrant, D. Meyer, N. Manno, M. DeMarchis, and M. G. Paoletti, "Current views on fungal chitin/chitosan, human chitinases, food preservation, glucans, pectins and inulin: a tribute to Henri Braconnot, precursor of the carbohydrate polymers science, on the chitin bicentennial," Carbohydrate Polymers, vol. 87, pp. 995-1012, 2012.

[18] K. E. Park, H. K. Kang, S. J. Lee, B. M. Min, and W. H. Park, "Biomimetic nanofibrous scaffolds: preparation and characterization of PGA/chitin blend nanofibers," Biomacromolecules, vol. 7, no. 2, pp. 635-643, 2006.

[19] H. K. Noh, S. W. Lee, J. M. Kim et al., "Electrospinning of chitin nanofibers: degradation behavior and cellular response to normal human keratinocytes and fibroblasts," Biomaterials, vol. 27, no. 21, pp. 3934-3944, 2006. 
[20] R. Murugan and S. Ramakrishna, "Nano-featured scaffolds for tissue engineering: a review of spinning methodologies," Tissue Engineering, vol. 12, no. 3, pp. 435-447, 2006.

[21] W. E. Teo and S. Ramakrishna, "A review on electrospinning design and nanofibre assemblies," Nanotechnology, vol. 17, no. 14, article R01, pp. R89-R106, 2006.

[22] N. Bhardwaj and S. C. Kundu, "Electrospinning: a fascinating fiber fabrication technique," Biotechnology Advances, vol. 28, no. 3, pp. 325-347, 2010.

[23] P. Sangsanoh and P. Supaphol, "Stability improvement of electrospun chitosan nanofibrous membranes in neutral or weak basic aqueous solutions," Biomacromolecules, vol. 7, no. 10, pp. 2710-2714, 2006.

[24] K. E. Park, S. Y. Jung, S. J. Lee, B. M. Min, and W. H. Park, "Biomimetic nanofibrous scaffolds: preparation and characterization of chitin/silk fibroin blend nanofibers," International Journal of Biological Macromolecules, vol. 38, no. 3-5, pp. 165-173, 2006.

[25] M. S. Kim, I. Jun, Y. M. Shin, W. Jang, S. I. Kim, and H. Shin, "The development of genipin-crosslinked poly(caprolactone) (PCL)/gelatin nanofibers for tissue engineering applications," Macromolecular Bioscience, vol. 10, no. 1, pp. 91-100, 2010.

[26] F. M. Hendriks, D. Brokken, C. W. J. Oomens, D. L. Bader, and F. P. T. Baaijens, "The relative contributions of different skin layers to the mechanical behavior of human skin in vivo using suction experiments," Medical Engineering and Physics, vol. 28, no. 3, pp. 259-266, 2006.

[27] C. Pailler-Mattei, S. Bec, and H. Zahouani, "In vivo measurements of the elastic mechanical properties of human skin by indentation tests," Medical Engineering and Physics, vol. 30, no. 5, pp. 599-606, 2008.

[28] S. G. Priya, H. Jungvid, and A. Kumar, "Skin tissue engineering for tissue repair and regeneration," Tissue Engineering B, vol. 14, no. 1, pp. 105-118, 2008.

[29] R. A. A. Muzzarelli, "Chitins and chitosans for the repair of wounded skin, nerve, cartilage and bone," Carbohydrate Polymers, vol. 76, no. 2, pp. 167-182, 2009. 

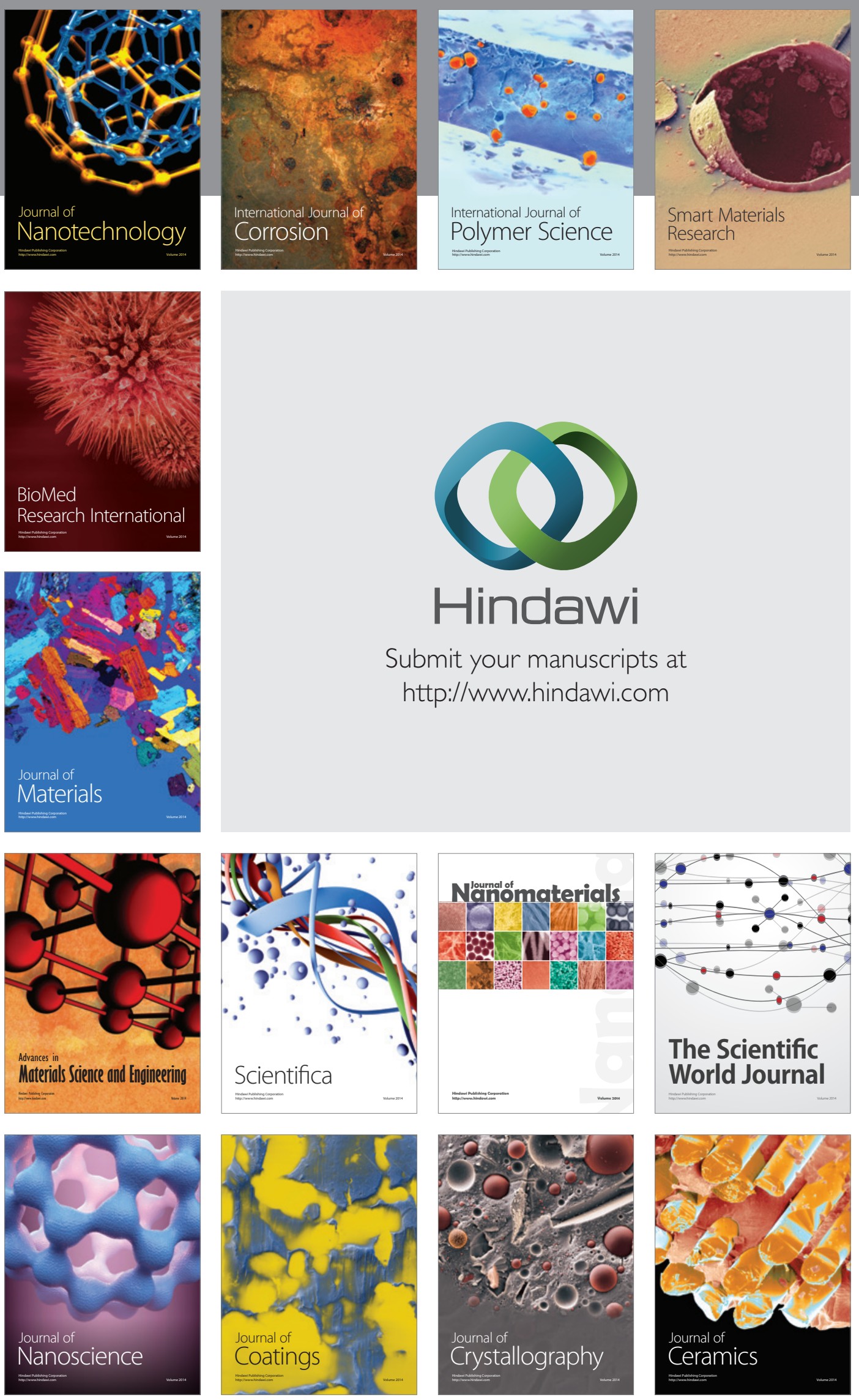

The Scientific World Journal

Submit your manuscripts at

http://www.hindawi.com

\section{World Journal}

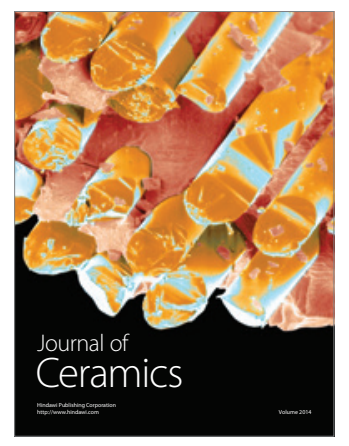

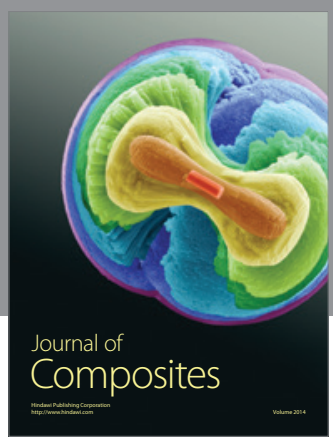
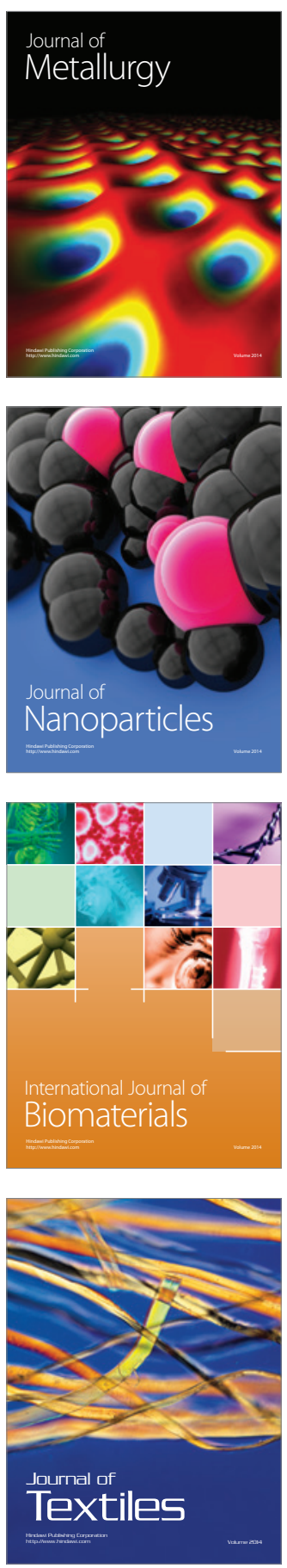